\title{
Optimization of the Merging Area After Toll
}

\author{
Li Lin ${ }^{1,2}$, Liang $\mathrm{Su}^{1,3}$, Shaozhen Chen ${ }^{1,3} \&$ Yuanbiao Zhang ${ }^{1}$ \\ ${ }^{1}$ Innovation Practice Base of Mathematical Modeling, Jinan University, Zhuhai, China \\ ${ }^{2}$ Business Administration Department of International Business School, Jinan University, Zhuhai, China \\ ${ }^{3}$ Finance Department of International Business School, Jinan University, Zhuhai, China \\ Correspondence: Li Lin, Administration Department of International Business School, Jinan University, \\ Qianshan Road 206\#, Zhuhai City, Guangdong Province, Post No. 519070, China. E-mail: \\ li1258085120@163.com
}

Received: November 22, 2017

Accepted: January 14, 2018 Online Published: January 17, 2018

doi:10.5539/emr.v7n1p17

URL: http://doi.org/10.5539/emr.v7n1p17

\begin{abstract}
In this paper, we focus on the planning of the merging area after toll and have established an optimization model by means of nonlinear integer programming model. With the consideration of factors such as safety, cost, and throughput, we select indexes including traffic flow, vehicle speed, lane width and the number of lanes to calculate and determine the tollbooth and merging area, and depending on the situation, we built modification model and reconstruction model. Then, we select Delaware Memorial Bridge toll plaza as an example to evaluate the effectiveness of our solutions based on the optimal model. We apply the method of computer simulation to solve the model, and we find that some parameters with the fixed value such as forced exchange rate, average expected speed and gradient rate may affect the applicability of the model. Then, we carry out the sensitivity analysis with the three parameters to determine the scope of application of the model. Finally, we further discuss development direction of this model.
\end{abstract}

Keywords: nonlinear integer programming model, computer simulation, sensitivity analysis

\section{Introduction}

With the sustained and rapid development of the global economic, individual car ownership is growing. The contradiction between the rapid growth of traffic and the limited traffic supply lead to traffic congestion and some traffic accidents, especially happening in the toll square. Thus, the issues about how to optimize the toll square to improve traffic congestion aroused the concern of all sectors of society

Existing research on toll system mainly uses the methods of queuing theory, regression analysis, and VISSIM simulation model and study on the service level indexes, traffic capacity and the optimal number of toll station. Gang Zhou and others reference M/G/K model to study the traffic capacity of toll station, and get the ideal results (Zhou \& Chang, 2001). Jun Xie built traffic model, based on the traffic behavior of the vehicles in toll station, and gave the calculation formula reflecting the service level index of toll station (Xie, 2007). Ming Zhang, Hong Chen and others applied the Regression Analysis to analyze the number of annual traffic accidents at the toll station, and a Safety Evaluation Model of toll station was established to predict the highway toll station of traffic accidents (Zhang, Chen, \& Wu, 2009). Wen Hang carried on the simulation analysis for the traffic capacity, vehicle queue delay and other service level indicators of toll station under different traffic volume and traffic composition (Hang, 2007). Shijian He used VISSIM simulation model to study the relationship of the traffic capacity of the mixed toll station with the total number of lanes and the proportion of ETC lanes, under the different arrival traffic and ETC traffic flow ratio (He, 2015). Jian Li studied the comprehensive traffic capacity of toll station at different proportions of artificial toll and ETC toll by using VISSIM simulation model ( $\mathrm{Li}$, Liu, Hu, Weng, \& Rong, 2012). Chuangxin Ding built the toll K/G/K model based on the analysis on traffic flow characteristics of highway toll station, and calculated the maximum level of service traffic toll at different service levels, based on in-depth analysis of field survey data (Ding \& Ceng, 2005). Yingming Guo built resource allocation model of highway toll station using random service theory and $\mathrm{M} / \mathrm{M} / \mathrm{m} / \infty / \infty / \mathrm{FCFS}$ queuing system model, and study algorithm of the optimal number of toll station (Guo, 2015). Quanru Pan optimized the existing queuing model with operational research theory, in view of the characteristics of toll station traffic flow, and study the optimal number of toll channels in M/M/m system (Pan, 2009). 
This paper focuses on the planning of the merging area after toll, analyzing the factors that affect the traffic passing efficiency and size of the merged area, and established Optimization Model of toll stations merge area depending on the nonlinear integer programming method to alleviate the problem of toll plaza congestion.

\section{Model Design}

Reference to the literature, a completed toll station is consisted by the toll channel, toll plaza, toll booths, and other facilities. And the toll channel includes toll booths, lanes, and other parts. As shown in Figure, the design of merging area is based on the design of the length, area and road gradient rate of merging area. Considering these factors, we first establish the indexes related to cost, safety and throughput, and then use the method of integer programming to design the modification solution and reconstruction solution.

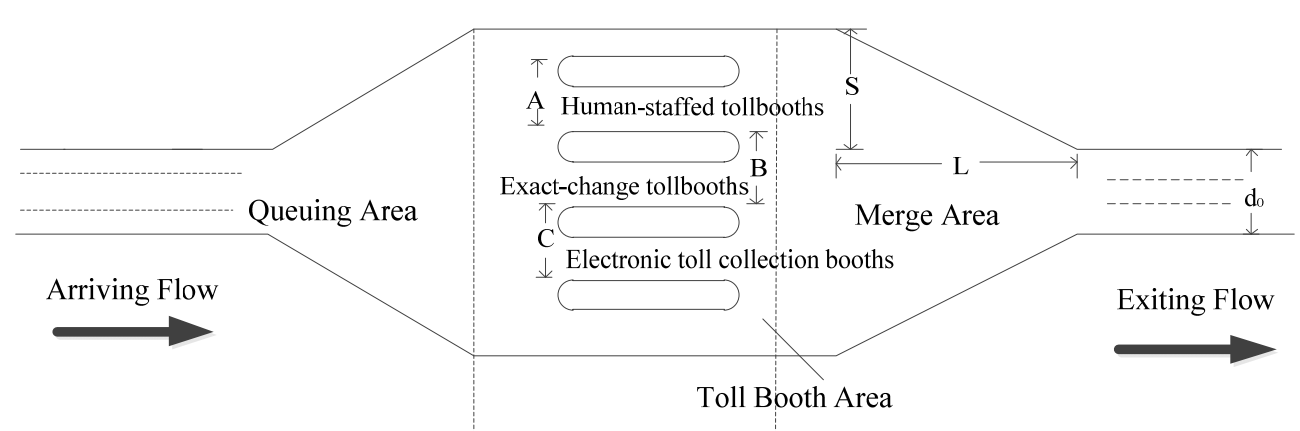

Figure 1. Layout of toll plaza

\subsection{Index of Cost, Safety and Throughput}

\subsubsection{Road gradient Rate}

The merging area of toll should meet the requirements to guide vehicles access to the toll plaza naturally. The number of traffic accidents is proportional to the road gradient rate of the toll plaza, so we limited the road gradient rate within a certain range when designing solutions. Reference to the literature, the road gradient rate and the number of traffic accidents in the toll plaza are in an exponential relationship.

$$
Y=1.4231 e^{8.0664 k} \text {; }
$$

Where, $Y$ is the number of traffic accidents and $k$ is the road gradient rate. The change of $Y$ is shown in Figure.

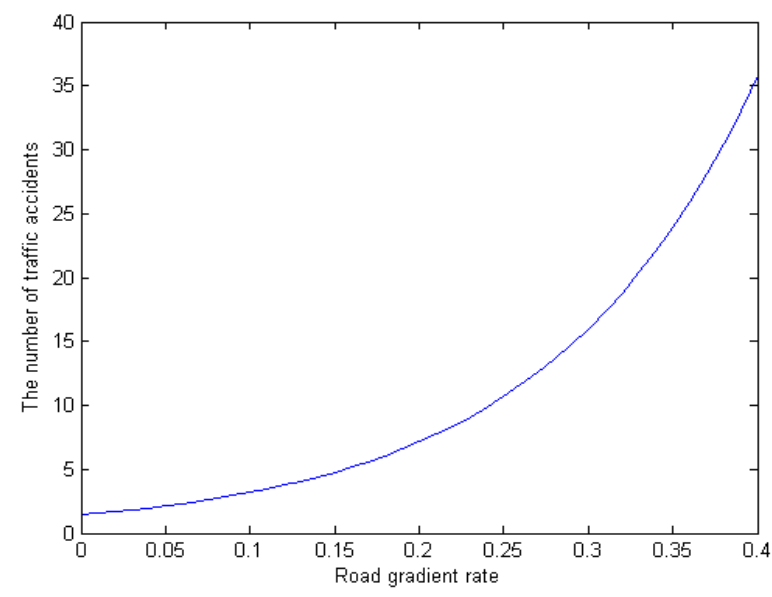

Figure 2. Relationship between traffic accidents and gradient rate

\subsubsection{Capacity of Bottleneck Road}

Multi-lane roadway close to the middle of the road lane is the main line, and away from the road center known is the side lane. The maximum road traffic is maximum when the flow of main and side lanes can provide sufficient free lane spacing. 


$$
\frac{q_{2}^{2}}{v_{2}{ }^{2}} \beta^{2}+\left(\frac{2 q_{1} q_{2}}{v_{1} v_{2}}-\frac{q_{2} k_{j}}{v_{2}}\right) \beta+\left(\frac{q_{1}^{2}}{v_{1}{ }^{2}}-\frac{q_{1} k_{j}}{v_{1}}\right)=\frac{3600 k_{j}}{t v_{f}} \ln (1-\beta) ;
$$

Where, $q_{1}, q_{2}$ are the flow of main and side lanes. $v_{1}, v_{2}$ are the average expected speed of main and sideline. $t$ is the minimum time headway in free lane changing. $\beta$ is the Forced exchange rate, $k_{j}$ is the maximum traffic density, $v_{f}$ is average speed without clogging. We get the capacity of Bottleneck road after we transformed Equation (3)

\subsection{Modification Model}

$$
Q=\left(2 v_{1} v_{2} \sqrt{\frac{1}{4} k_{j}^{2}+\frac{3600 k_{j}}{t v_{f}} \ln (1-\beta)}+k_{j} v_{1} v_{2} /\left(v_{2}+\beta v_{1}\right) ;\right.
$$

In real life, people often simply need to adjust the toll plaza. In order to ensure cost, safety, efficiency to achieve the optimization. Based on Nonlinear Integer Programming method, Modification model is built to enhance capacity through making adjustments to the proportion of tollbooths, size and shape of merging area after toll. Therefore a complete solution is proposed.

\subsubsection{Constraint of Throughput}

In order to avoid congestion at the toll plaza, the throughput is required to be less or equal to the capacity of bottleneck road.

$$
q=a x_{1}+b x_{2}+c x_{3} \leq Q ;
$$

Where, $a, b, c$ is the capacity of human-staffed tollbooths, exact-change tollbooths, electronic toll collection booths. $x_{1}, x_{2}, x_{3}$ is the number of human-staffed tollbooths, exact-change tollbooths, electronic toll collection booths. $Q$ is the capacity of bottleneck road. $q$ is the throughput.

\subsubsection{Constraint of Safety}

To make the toll plaza to meet the safety standards, requiring the toll plaza Road gradient limited to $1 / 5$ to 1/7.From the geometric relationship, we get the equation of $\mathrm{K}$ is:

$$
k=\frac{S}{L}=\frac{A x_{1}+B x_{2}+C x_{3}-d_{0}}{2 l} ;
$$

Where, $A, B, C$ is the width of human-staffed tollbooths, exact-change tollbooths and electronic toll collection booths. $d_{0}$ is the width of freeway connected with the toll. $l$ is the merging area length. The road gradient is limited between $1 / 5$ and $1 / 7$

$$
\frac{1}{5} \leq k \leq \frac{1}{7}
$$

\subsubsection{Constraint of Cost}

Besides the constraint of through and safety, we also considered the constraint of cost when we design modification solution. Costs include the cost of construction of various tollbooths and the cost of expansion or demolition of the toll plaza. Cost of construction of various tollbooths $c_{1}$ is

$$
c_{1}=\alpha x_{1}+\beta x_{2}+\gamma x_{3}
$$

Where, $\alpha, \beta, \lambda$ are the cost of construction of human-staffed tollbooths, exact-change tollbooths and electronic toll collection booths. Cost of expansion or demolition of the toll plaza $c_{2}$ is

$$
c_{2}=\Delta s \times c_{0} ;
$$

Where, $\Delta s$ is the change of merging area and $c_{0}$ is the cost of per area. The total cost is

$$
c=c_{1}+c_{2} \text {. }
$$

\subsubsection{Other Constraints}

When we change the original toll station, the total number of toll channel is constant.

$$
x_{1}+x_{2}+x_{3}=n_{0}
$$

Where, $n_{0}$ is the total number of toll channel.

\subsubsection{Modification Solution based on Integer Programming}

From the above conditions, the inter programming model for modification solution is: 


$$
\begin{aligned}
& \min \quad c \\
& \left\{\begin{array}{l}
a x_{1}+b x_{2}+c x_{3} \leq Q \\
\frac{1}{5} \leq \frac{A x_{1}+B x_{2}+C x_{3}-d_{0}}{2} \leq \frac{1}{7} \\
x_{1}+x_{2}+x_{3}=n_{0} \\
0 \leq x_{i} \leq n_{0} \quad(i=1,2,3) \\
0 \leq l
\end{array} .\right.
\end{aligned}
$$

\subsection{Reconstruction Model}

In real life, Reconstruction model is applied when a new toll plaza is designed or rebuilt. To ensure cost, safety, efficiency to achieve the optimization. Based on Nonlinear Integer Programming method, Reconstruction model is built to enhance capacity through adjusting the number and proportion of tollbooths, size and shape of merging area after toll. Therefore, a complete solution is proposed. Constraints of the model are similar to above instructions. The cost of rebuilding has to be especially emphasized. The cost involved not only toll booths but also the area to be built and other costs of construction.

From the above conditions, the inter programming model for reconstruction solution is

$$
\begin{aligned}
& \min \quad c \\
& \left\{\begin{array}{l}
a x_{1}+b x_{2}+c x_{3} \leq Q \\
\frac{1}{5} \leq \frac{A x_{1}+B x_{2}+C x_{3}-d_{0}}{2} \leq \frac{1}{7} \\
0 \leq x_{i} \leq n_{0} \quad(i=1,2,3) \\
0 \leq l
\end{array} .\right.
\end{aligned}
$$

\section{Evaluation and Comparison of Solutions}

In this part, we combine the date of Delaware Memorial Bridge toll plaza with our model to propose modification and reconstruction solutions. Then we compare three indexes such as average wasted time, cost and safety in the three solutions to determine the effectiveness of the model.

\subsection{Establishment of Traffic Capacity Evaluation Index}

In order to evaluate the effectiveness of the model, original solution, modification solution and reconstruction solution should be compared through an indicator. A vehicle traveling in a lane has to wait or slow down to allow another vehicle in the adjacent lane to pass. These kinds of delays are termed as merging delays.

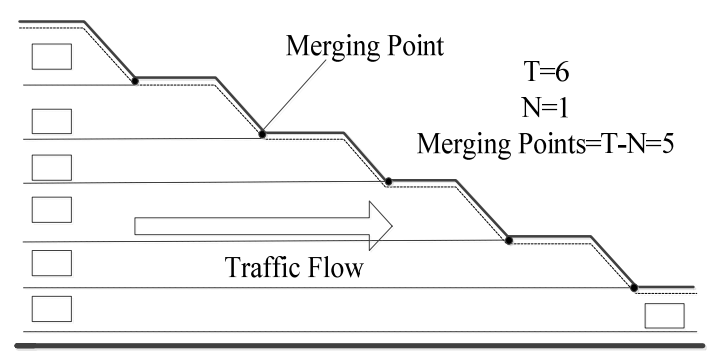

Figure 3. Merging points and conflicting lanes

We use the average wasted time to measure the merging delays. The time wasted at merging area is calculated using the following logic. When vehicles exit from the tollbooths $T$, and then finally merge into $\mathrm{N}$ lanes of the highway. As a result, the numbers of merging points is given by $(T-N)$ from Figure, we can see that in a toll plaza with aside merging layout which has T tollbooths, the first merging point takes a stream coming from 2 tollbooths and the second merging point would take a stream from 3 . With Little's theorem, the average waiting time in the system is given by the Equation (12). 


$$
t_{s y s}(\lambda)=\frac{L(\lambda)}{\lambda}=\frac{1}{\mu_{B}-\lambda}+\frac{\mu_{B}-\mu_{0}}{\lambda\left(\mu_{B}-\mu_{0}\right)+\mu_{0} \mu_{B}} ;
$$

Average wasted time at each merging point is the difference between $t_{\text {sys }}$ and the time driver would spend on a normal lane. The expected time a driver spends when no merging happens is $1 / \mu_{0}$. Therefore, average wasted time is given by the Equation (13).

$$
t_{\text {diff }}(\lambda)=\operatorname{tsys}(\lambda)-\frac{1}{\mu_{0}}=\frac{1}{\mu_{B}-\lambda}+\frac{\mu_{B}-\mu_{0}}{\lambda\left(\mu_{B}-\mu_{0}\right)+\mu_{B} \mu_{0}}-\frac{1}{\mu_{0}} ;
$$

Where $\mu_{B}$ is the service rate when merging happens ( $\mathrm{pcu} / \mathrm{hr}$ ), $\mu_{0}$ is the service rate when no merging happens $(\mathrm{pcu} / \mathrm{h}), \lambda$ is the vehicle arrival rate, $\lambda=(k / T) \times \phi(\mathrm{pcu} / \mathrm{h}), k=$ No. of conflicting lanes at each merging point and it has a range between 2 and $T-N+1$.

The above equation gives the average wasted time of a driver at each merging point. The total average wasted time $(\bar{W})$ can be calculated using the Equation (14).

$$
\bar{W}=\sum_{i=1}^{T-N} \frac{i+1}{T} \times t_{\text {diff }}\left(\frac{i+1}{T} \times \phi\right) \mathrm{sec} / v e h ;
$$

Where $\bar{W}$ is the total average wasted time, $i$ is No. of merging points, $t_{\text {diff }}$ is the average wasted time at No. $i$ merging point, $\phi$ is the traffic flow.

As the safety and cost are fully considered in the model. By comparing the length of average wasted time, the effectiveness of the different solutions can be measured perfectly. The shorter average wasted time is, the better the solution is.

\subsection{Comparison of Solutions}

As is known to us, the traffic conditions are terrible around the Delaware Memorial Bridge toll. Therefore, the modification solution and reconstruction solution can be applied by using our above model. Specific solutions design and traffic evaluation are as follows in Table 1.

Table 1. Solution design and traffic condition evaluation

\begin{tabular}{llll}
\hline & $\begin{array}{l}\text { Original } \\
\text { Solution }\end{array}$ & $\begin{array}{l}\text { Modification } \\
\text { Solution }\end{array}$ & $\begin{array}{l}\text { Reconstruction } \\
\text { Solution }\end{array}$ \\
\hline $\begin{array}{l}\text { Number of human-staffed tollbooths } \\
\text { Number of Exact-change tollbooths }\end{array}$ & 2 & 1 & 1 \\
$\begin{array}{l}\text { Number of Electronic toll collection } \\
\text { booths }\end{array}$ & 4 & 3 & 4 \\
Merging area length & $59 \mathrm{~m}$ & 8 & 4 \\
Road gradient rate & 0.2 & $110.4 \mathrm{~m}$ & $87.7 \mathrm{~m}$ \\
Security level & Average & 0.135 & 0.125 \\
Accidents per month & 11 & Good & Excellent \\
Merging area after toll & $1663.8 \mathrm{~m}^{2}$ & 7 & 4 \\
Average wasted time & $10.09 \mathrm{~s}$ & $5431 \mathrm{~m}^{2}$ & $765 \mathrm{~m}^{2}$ \\
Traffic Volume & $9500 \mathrm{PCU} / \mathrm{h}$ & $6.8725 \mathrm{~s}$ & 2.5523 \\
Total cost & $/$ & $9500 \mathrm{PCU} / \mathrm{h}$ & $9500 \mathrm{PCU} / \mathrm{h}$ \\
\hline
\end{tabular}

As shown in Table 1, the modification solution adjusts to the proportion of various types of tollbooths, the merging area length and size. As a result, the average wasted time is greatly reduced, and the efficiency is largely improved. In terms of safety, by reducing the grading rate, the security level is up to "good" level. The reconstruction solution proposes to reduce the number of toll booths and gradient rate. As a result, though the cost is very high, the traffic efficiency and security level are all optimal. 


\subsection{Conclusions of the Two Models}

Regarding traffic congestion and security issues in toll plaza of New Jersey Turnpike, the modification and reconstruction model are both available. And the cost and security level of reconstruction are higher than the modification model's, which is in line with the real situation. The modification model and reconstruction model are effective.

\section{Sensitivity Analysis}

In our model, there are some parameters with a fixed value. By changing them, we can study their impact on the model to better regulate the actual situation.

\subsection{Sensitivity of Forced Exchange Rate in Reconstruction Model}

The forced exchange rate is the probability that the vehicles are forced to change lanes due to congestion or obstruction. Because driver blind to follow the rapid flow and constantly change lanes when congestion, force exchange rate will be changed frequently. And our model determines its value. Therefore, it's necessary to carry out the sensitivity analysis on it. Because the maximum traffic flow is a constant in the same road, the sensitivity analysis should be carried out in a fixed maximum traffic flow in reconstruction model.

Table 2. Sensitivity of forced exchange rate

\begin{tabular}{lllllll}
\hline $\begin{array}{l}\text { Forced } \\
\text { exchange rate }\end{array}$ & $\begin{array}{l}\text { Road } \\
\text { gradient rate }\end{array}$ & $\begin{array}{l}\text { Merging } \\
\text { Length } \\
(\mathrm{m})\end{array}$ & $\begin{array}{l}\text { Merging } \\
\text { Area } \\
\left(\mathrm{m}^{2}\right)\end{array}$ & $\begin{array}{l}\text { human-staffed } \\
\text { tollbooths }\end{array}$ & $\begin{array}{l}\text { exact-change } \\
\text { tollbooths }\end{array}$ & $\begin{array}{l}\text { ETC } \\
\text { booths }\end{array}$ \\
\hline 0.5 & 0.127 & 89.4 & 763.2 & 1 & 4 & 2 \\
0.6 & 0.125 & 90.9 & 765.4 & 1 & 4 & 2 \\
0.7 & 0.122 & 92.2 & 766.5 & 1 & 4 & 2 \\
\hline
\end{tabular}

As shown in Table 2, the change in forced exchange rate has slight influence in the road gradient rate, length and area, and has no effect on the proportion of tollbooths.

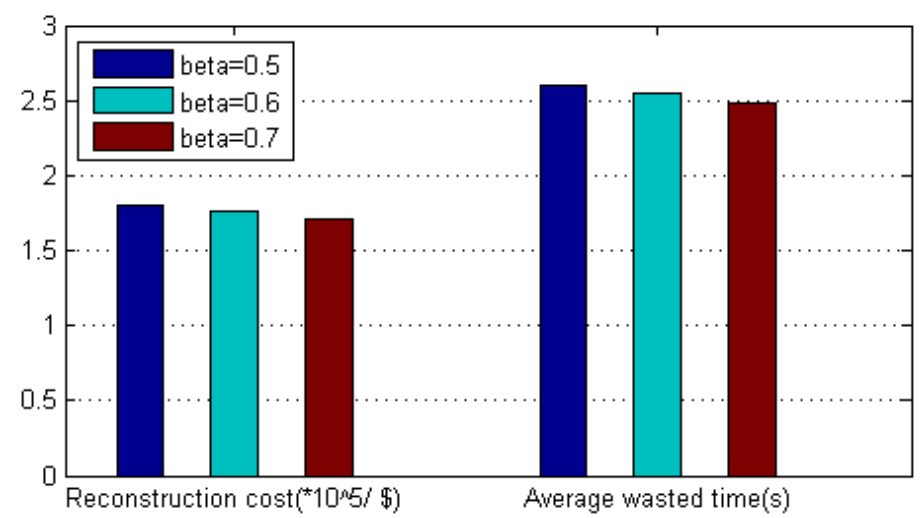

Figure 4. Cost and average wasted time at different forced exchange rate

According to Figure, we can find that the change of cost and average wasted time are small, which indicates that the model has low sensitivity to the forced exchange rate. Since the merging area is a fan-shaped area, there must have the certain range for it. However, the higher the forced exchange rate is, the greater the security risk is. Thus, the forced exchange rate has to be set in a certain range.

In short, the force exchange rate has little effect on the model, and the model can be applied in lots of condition. But considering safety, it still should be controlled in a certain range.

\subsection{Sensitivity of Average Expected Speed in Reconstruction Model}

We treat the average expected speed as a constant while modeling. However, it will vary according to the actual 
situation in fact. Therefore, we made a sensitivity analysis of it.

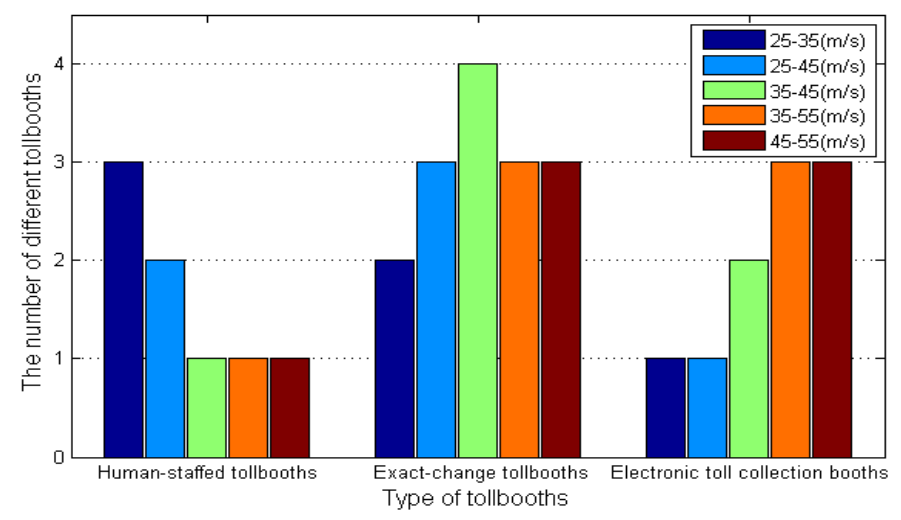

Figure 5. the proportion of toll stations at different average expected speed

As shown in Figure, with the increase of the average expected speed, the number of human-staffed tollbooths decreased, the exact-change tollbooths fluctuate rise, and the electronic toll collection booths increased.

Table 3. Sensitivity analysis of average expected speed

\begin{tabular}{lllllll}
\hline $\begin{array}{l}\text { Average expected } \\
\text { speed of the main } \\
\text { line }(\mathrm{m} / \mathrm{s})\end{array}$ & $\begin{array}{l}\text { Average } \\
\text { speed } \\
\text { sideline(m/s) }\end{array}$ & $\begin{array}{c}\text { expected } \\
\text { of }\end{array}$ & $\begin{array}{l}\text { Maximum the } \\
\text { flow(pcu/h) }\end{array}$ & $\begin{array}{l}\text { Road gradient } \\
\text { rate }\end{array}$ & $\begin{array}{l}\text { Merging } \\
\left.\text { Area(m }{ }^{2}\right)\end{array}$ & $\begin{array}{l}\text { Merging } \\
\text { Length }\end{array}$ \\
\hline 35 & 25 & 2885.0 & 0.126 & 764.40 & 87.32 \\
45 & 25 & 3281.3 & 0.125 & 765.42 & 87.54 \\
45 & 35 & 3852.9 & 0.125 & 765.45 & 87.70 \\
55 & 35 & 4293.6 & 0.122 & 765.49 & 87.82 \\
55 & 45 & 4812.6 & 0.120 & 765.53 & 88.00 \\
\hline
\end{tabular}

Table shows that when the average expected speed increase, maximum traffic flow, and the area as well as length of merging area increased slightly, and the road gradient rate decreases, among which the change of the road gradient rate, area and length are small.

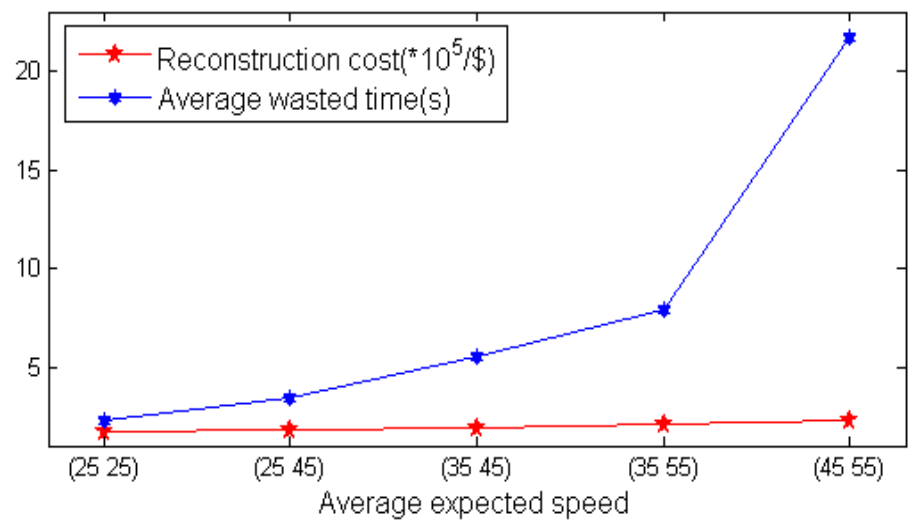

Figure 6. The proportion of toll stations at different average expected speed

As shown in Figure 6, both are on the rise, but the change is small in the cost and large in the average wasted 
time. So, the model is sensitivities to the average expected speed.

According to the Equation 1, to ensure safety at an excellent level, the gradient rate should be in a certain range. Meanwhile, because of the cost, the number of toll station change little, so that the change of merging area is little as well. However, the increase of average expected speed leads to the significant increase in traffic volume. Thus, the change of the maximum capacity of the road is smaller than the change of the traffic volume, which causes the congestion and the increase of average wasted time. Therefore, considering the safety, it is necessary and reasonable to limit the average expected speed in a certain range.

\subsection{Sensitivity of Gradient Rate in Modification Model}

In our modification model, to simplify the model and considering the safety, we have limited the variable range of the gradient rate, which also has the influence on the shape of tollbooths. Thus, in this section, we will conduct a sensitivity analysis to study the effect of gradient rate on the model result.

Table 4. The sensitivity of gradient rate

\begin{tabular}{lrllllll}
\hline $\begin{array}{l}\text { Change } \\
\text { gradient } \\
\text { range }\end{array}$ & $\begin{array}{r}\text { of } \\
\text { rate }\end{array}$ & $\begin{array}{l}\text { Gradient } \\
\text { rate }\end{array}$ & $\begin{array}{l}\text { Merging } \\
\text { Area } \\
\left(\mathrm{m}^{2}\right)\end{array}$ & $\begin{array}{l}\text { Merging } \\
\text { Length } \\
(\mathrm{m})\end{array}$ & $\begin{array}{l}\text { Human-staffed } \\
\text { tollbooths }\end{array}$ & $\begin{array}{l}\text { Exact-change } \\
\text { tollbooths }\end{array}$ & $\begin{array}{l}\text { Electronic toll } \\
\text { collection } \\
\text { booths }\end{array}$ \\
\hline $1 / 3-1 / 5$ & 0.20 & 3394.8 & 69 & 1 & 3 & 8 \\
$1 / 5-1 / 8$ & 0.12 & 5431.7 & 110.4 & 1 & 3 & 8 \\
$1 / 12-1 / 18$ & 0.08 & 8147.5 & 165.6 & 1 & 3 & 8 \\
\hline
\end{tabular}

As shown in Table 4, the change of gradient rate range does not affect the proportion of tollbooths, and the area and length of the merging area change in the same direction as the gradient rate.
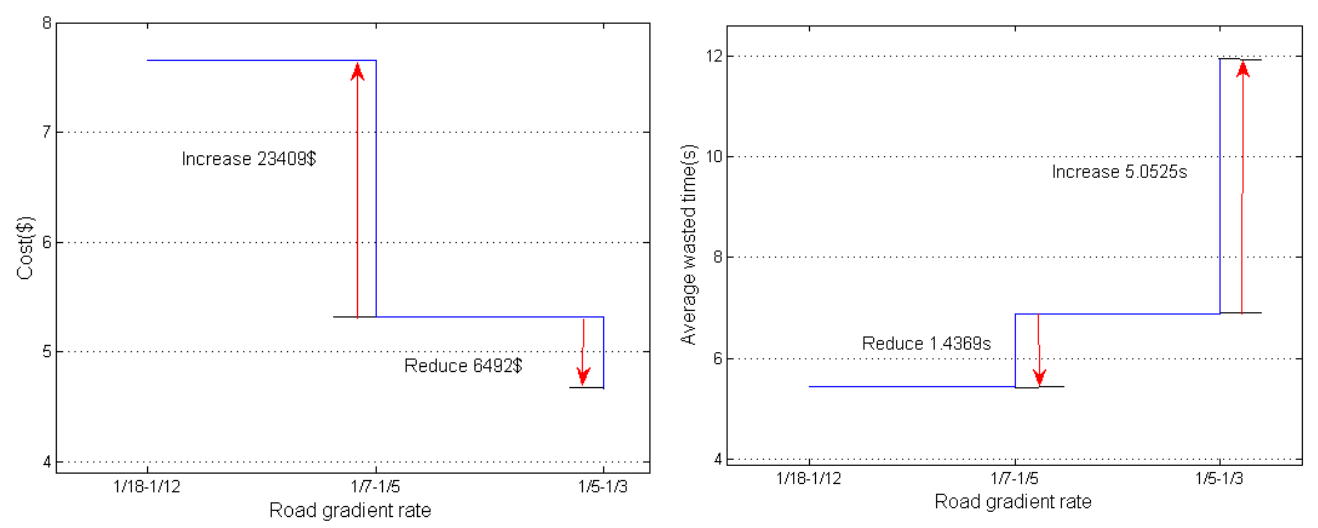

Figure 7. The cost and average wasted time in different gradient rate

As shown in Figure 7, the change or gradient rate has great influence on the cost and average wasted time. Combine with Figure and Figure, the reducing in safety level cannot save the cost well, and the average wasted time increase significantly. Also, when we reduce the gradient rate to achieve a higher safety level, the minor change of traffic accident will significantly improve cost, and the average wasted time change minor as well.

The gradient level has a greater impact on the model. But considering the security problem and the relationship between output as well as input, the gradient rate should be controlled in a certain level.

\section{Conclusions}

In this paper, we establish Optimization Model of the toll stations merging area on the basis of the nonlinear integer programming method. Because vehicle combination and toll plaza congestion are affected and determined by merging area and toll composition, that's mean different size and shape of merging area should correspond to the different number and proportion of toll, so we make a modeling analysis of merging area and 
the number and type of tolls. We determine the size, shape and pattern of the merging area after toll by planning the merging length, merging area, gradient rate and the number and type of tollbooths. During the process, actual traffic flow, vehicle speed, lane width and other indicators are taken into consideration. Depending on the situation, we divide the optimization model into modification model and reconstruction model.

We select Delaware Memorial Bridge toll plaza on New Jersey Turnpike as an example, choose the total cost, the average delay time of vehicles, and the security level as the standard to compare the current scheme, modification solution and reconstruction solution, and prove the effectiveness and reliability of the model. Meanwhile, we find that the change of some evaluation of fixed parameters, such as the force exchange rate, average expected speed and gradient rate, makes the result unstable, so we carry out the sensitivity analysis with these three factors. The result shows that the change of these three factors result in a large change in the results and the factors should be controlled within a small rang. Based on the actual situation in the security level range, it still proves that the models have stability and universal applicability.

\section{Acknowledgments}

While remaining responsible for any errors in this paper, the authors would like to thank guidance and advice of Dr. Yuanbiao Zhang preparing the article.

\section{References}

Bing, L. (2008). Research on expressway electronic toll collection system simulation and application. Application Research of Computers, 25(6), 1914-1916.

Chuangxin, D., \& Yingkun, Z. (2005). Analysis of traffic capacity of high-grade highway toll station. Journal of Kunming University of Science and Technology (Natural Science Edition), (06), 90-93+104.

Gang, Z., \& Chengli, C. (2001). Study on the traffic capacity of highway toll station. Journal of Highway and Transportation Research and Development, (03), 56-59.

Gu, L. (2010). Analysis on expressway toll capacity. Highway Engineering, 35(3), 153-155.

Hongwei, S. (2006). Mathematical model analysis of vehicle queuing at expressway toll station. Science and Technology of West China, (16), 34-35.

Jian, L., Lili, L., Bin, H., Jiancheng, W., \& Jian, R. (2012). Simulation - based method for determining the mix of freeway traffic capacity of toll stations. Highway, (07), 183-188.

Jr, M. D., \& Jr, R. E. S. (2001). Contribution to the development of guidelines for toll plaza design. Journal of Transportation Engineering, 127(3), 215-222.

Jun, X. (2007). The expressway capacity analysis and research of service quality evaluation. Chang'an University.

Komada, K., \& Nagatani, T. (2010). Traffic flow through multi-lane tollbooths on a toll highway. Physica A Statistical Mechanics \& Its Applications, 389(11), 2268-2279. https://doi.org/10.1016/j.physa.2010.01.041

Komada, K., Masukura, S., \& Nagatani, T. (2009). Traffic flow on a toll highway with electronic and traditional tollgates. Physica A Statistical Mechanics \& Its Applications, 388(24), 4979-4990. https://doi.org/10.1016/j.physa.2009.08.019

Lin, F. B., \& Su, C. W. (1994). Level-of-service analysis of toll plazas on freeway main lines. Journal of Transportation Engineering, 120(2), 246-263. https://doi.org/10.1061/(ASCE)0733-947X(1994)120:2(246)

Ming, Z., Hong, C., \& Xiaowu, W. (2009). Safety Evaluation Model of highway toll station. China Safety Science Journal, 19(10), 139-144+179.

Quanru, P. (2009). Application of queuing theory in toll station design and management. Operations Research Transactions, 13(3), 95-102.

Shijian, H. (2015). Simulation of traffic capacity of expressway mixed toll station. Journal of Transport Science and Engineering, 31(3), 106-111.

Su. (2012). Study on Design of Toll Station of Expressway. Changan University.

Wei, D., Haixiang, W., \& Wei, W. (2000). Study on the capacity of highway toll entrance. China Journal of Highway and Transport, 13(2), 87-90.

Wen, H. (2007). Study on capacity of highway toll station based on simulation. Journal of Transport Information and Safety, 25(6), 60-62. 
Yingming, G. (2015). Highway toll station Research on Optimization of resource allocation based on lane queuing system model. Value Engineering, 34(11), 198-201.

Zhiyong, Z. (2001). Study on expressway toll capacity in Beijing area based on M/G/K queuing model. Highway, (7), 128-133.

\section{Copyrights}

Copyright for this article is retained by the author(s), with first publication rights granted to the journal.

This is an open-access article distributed under the terms and conditions of the Creative Commons Attribution license (http://creativecommons.org/licenses/by/4.0/). 